\title{
Effective Use of Focused Ion Beam (FIB) in Investigating Fundamental Mechanical Properties of Metals at the Sub-Micron Scale
}

\author{
Julia R. Greer \\ EMDL, PARC, 3333 Coyote Hill Road, Palo Alto, CA, 94304
}

\begin{abstract}
Recent advances in the 2-beam focused ion beams technology (FIB) have enabled researchers to not only perform high-precision nanolithography and micro-machining, but also to apply these novel fabrication techniques to investigating a broad range of materials' properties at the submicron and nano-scales. In our work, the FIB is utilized in manufacturing of sub-micron cylinders, or nano-pillars, as well as of TEM cross-sections to directly investigate plasticity of metals at these small length scales. Single crystal nano-pillars, ranging in diameter between 300 $\mathrm{nm}$ and $870 \mathrm{~nm}$, were fabricated in the FIB from epitaxial gold films on $\mathrm{MgO}$ substrates and subsequently compressed using a Nanoindenter fitted with a custom-fabricated diamond flat punch. We show convincingly that flow stresses strongly depend on the sample size, as some of our smaller specimens were found to plastically deform in uniaxial compression at stresses as high as $600 \mathrm{MPa}$, a value $\sim 25$ times higher than for bulk gold. We believe that these high strengths are hardened by dislocation starvation. In this mechanism, once the sample is small enough, the mobile dislocations have a higher probability of annihilating at a nearby free surface than of multiplying and being pinned by other dislocations.
\end{abstract}

Contrary to this, if the dislocations are trapped inside the specimen by a coating, the strengthening mechanism is expected to be different. Here we present for the first time the comparison of plastic deformation of passivated and unpassivated single crystal specimens at the sub-micron scale. The role of free surfaces is investigated by comparing stress results of both asFIB'd, annealed, and alumina-passivated pillars. Preliminary results show that ALD-coated pillars exhibit much higher flow stresses at equivalent sizes and strains compared with the uncoated samples. We also found that while FIB damage during pillar fabrication might account for a small portion of the strength increase, it is not the major contributor.

\section{INTRODUCTION}

In the last decade or so, the Focused Ion Beam has become one of the most widely used tools for sample fabrication, testing, and imaging[1],[2],[3]. This multidisciplinary tool is applicable to a broad range of projects like fabrication and testing of MEMS, sensors, microactuators, microfluidic devices, etc. With some care, FIB can be utilized in fabrication of intricate 3-D structures by both etching of the starting material and by deposition of metals from gas phase. One of the key contributions of FIB's availability for mechanical experiments is that it bridges the experimental length scale with the computational results, where sample sizes are generally specified in a number of atoms [4]. One of the areas where the community has benefited greatly from using the FIB is mechanics. Until recently, mechanical deformation has largely been carried out in thin films due to their relative ease of deposition and their industrial relevance. The development of the Focused Ion Beam (FIB) as a user-accessible fabrication tool 
has enabled constraining the specimens not only vertically but also in the in-plane directions. For example, an increasing number of research groups are studying size effects in plasticity by investigating fundamental mechanical deformation in micron- and sub-micron-sized pillars[5],[6],[7],[8]. Although a unified theory explaining plasticity below a certain length scale remains a matter of great research and controversy, the results of most computational and experimental studies indicate an inevitable strength increase associated with the reduction in sample size, or a so-called size effect.

\section{UNIAXIAL COMPRESSION EXPERIMENTS}

In this work, we focus our attention on size effects arising in uniaxial deformation of gold nano-pillars with and without passivation on $\mathrm{MgO}$ substrates. The FIB is instrumental in nearly every step of these experiments, starting with pillar fabrication, micro-machining of the flatpunch indenter tip, and ending with machining TEM samples for dislocation activity investigation. The compression is conducted in the DCM module of the MTS Nanoindenter, where a uniform top load is applied to the single crystalline sample of sub-micron dimensions, whose initial dislocation density is on the order of $10^{12} / \mathrm{m}^{2}$. This particular type of deformation is chosen because the material is not expected to strengthen through one of the known mechanisms like strain gradient plasticity, dislocation confinement, grain size hardening, or initial lack of dislocations. The uniaxial compression procedure and methodology for stress-strain calculations, as well as the FIB fabrication technique are described in detail elsewhere[9]. An example of gold pillar on $\mathrm{MgO}$ substrate is shown in Figure 1.

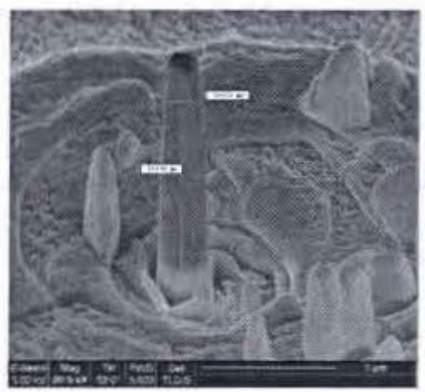

Figure 1. 290-nm diameter single crystal gold pillar fabricated in the FIB on $\mathrm{MgO}$ substrate.

The experiments presented here are unique in their attempt to assess the effects of surfaces on the pillar strength. While passivation of thin films is done routinely via sputtering or evaporation, uniform coating of complex geometries is much more challenging. The availability of Atomic Layer Deposition (ALD) for high-aspect ratio features is complementary to the FIB fabrication technique as it allows for studying the effects of passivation on the features of interest Key aspect of ALD is its utilization of sequential precursor gas pulses to deposit a film one layer at a time. The first precursor gas is introduced into the process chamber to produce a monolayer of gas on the substrate surface. The second precursor gas is then introduced into the chamber to react with the first gas on the surface of the substrate and to produce exactly a monolayer of film. Since each pair of gas pulses produces exactly one monolayer of film, the thickness can be precisely controlled. 


\section{DISCUSSION}

The results of our uniaxial compression experiments indicate a strong size effect: the flow stresses for gold pillars are much higher than the typical strength of bulk gold, estimated at 20 $\mathrm{MPa}$ at $2 \%$ strain. Moreover, the flow stresses for single-crystalline $<001>$-oriented pillars increase from $\sim 50 \mathrm{MPa}$ to $600 \mathrm{MPa}$ as the diameter is reduced from $870 \mathrm{~nm}$ to $300 \mathrm{~nm}$. Figure 2(a) shows the typical stress-strain curves for several uncoated nano-pillars, whose diameters varied from $300 \mathrm{~nm}$ to $870 \mathrm{~nm}^{\prime}$.

The methodology for stress calculation from the Nanoindenter-generated data is presented in detail elsewhere [9] 